
\title{
25 Research Soure \\ Peritoneal Tuberculosis in Children Over the Last Decade: A Systematic Literature Review
}

Inga Dekeryte ( $\nabla$ inga.dekeryte@fc.Ismuni.It )

Lithuanian University of Health Sciences Kaunas Clinics https://orcid.org/0000-0003-2613-7390

Ausra Lukosiute-Urboniene

LIthuanian University of Health Sciences

Kamile Donielaite-Anise

Lithuanian University of Health Sciences

Arturas Kilda

Lithuanian University of Health Sciences

Robertas Bagdzevicius

Lithuanian University of Health Sciences

Vidmantas Barauskas

Lithuanian University of Health Sciences

Research article

Keywords: peritoneal tuberculosis, abdominal tuberculosis, children, paediatric surgery

Posted Date: February 19th, 2021

DOI: https://doi.org/10.21203/rs.3.rs-244806/v1

License: (c) (i) This work is licensed under a Creative Commons Attribution 4.0 International License.

Read Full License 


\section{Abstract}

Background: Tuberculosis remains a public health issue. Peritoneal tuberculosis is an uncommon form of tuberculosis and it accounts for less than $1 \%$ of all tuberculosis cases in children. The aim was to asses experience and struggles in diagnosing peritoneal tuberculosis in children.

Methods: Systematic review of literature from the PubMed database was conducted including cases of children with peritoneal tuberculosis during 2008-2019 years.

Results: The search yielded 2309 potentially relevant articles, 11 met inclusion criteria with total number of 48 patients (4 our cases, 44 from the literature). 29 (60.4\%) were males with the median age of 11 years and $19(39.6 \%)$ females with the median age of 10 . The contact with active tuberculosis was reported in 16 cases (33.3\%). Tuberculin skin test was performed in 42 cases (87.5\%) with only $43.7 \%$ positive. The mean time from symptoms presentation until hospitalization was 30.6 days. The most common symptoms were abdominal pain (70.8\%), abdominal distention (66.7\%) and fever $(54.2 \%)$. The most common ultrasound findings were free fluid in pelvic cavity $(70.8 \%)$ and enlarged mesenteric lymph nodes (52.1\%). The majority of culture for Mycobacterium tuberculosis samples were negative. Surgery was performed in $36(75 \%)$ cases with the most common findings of tubercles $(63.8 \%)$, peritonitis $(41.6 \%)$ and ascites (36.1\%). Histopathological biopsy evaluation was performed in $38(79.2 \%)$ patients and in all cases tuberculosis granulomas were confirmed.

Conclusions: Clinical presentation, laboratory and radiological findings are nonspecific for peritoneal tuberculosis in children. This systematic review shows that peritoneal tuberculosis was diagnosed by surgical intervention in most of the cases. Accordingly, we suggest the algorithm of less invasive procedures and tests before surgical intervention.

\section{Background}

Tuberculosis (TB) is a chronic, infectious granulomatous disease caused by bacteria called Mycobacterium tuberculosis. According to the World Health Organization (WHO), about one-third of the world's population is infected by Mycobacterium Tuberculosis [1]. It is also one of the leading cause of death worldwide [1]. The bacilli Calmette-Guérin (BCG) vaccine has been shown to prevent severe forms of TB in children [4]. Unfortunately, BCG vaccine does not prevent from primary infection, neither reactivation of latent pulmonary infection. According to WHO, 10.0 million people globally fell ill with TB, of these $12 \%$ were children (under age $<15$ ) in 2019 [3].

There are 2 main forms of tuberculosis: pulmonary and extrapulmonary. Pulmonary TB is the most common form accounting for $80 \%$ of all TB cases. When TB affects other organs instead of lungs, it is called extrapulmonary TB. It might be abdominal, miliary, genitourinary tract, pleural, tuberculous meningitis, tuberculous lymphadenitis and other. Abdominal tuberculosis can affect gastrointestinal tract and peritoneum [2]. It is a very rare form with the incidence between $0.1-0.7 \%$ among all TB forms and mostly observed in adult patients [2]. Peritoneal tuberculosis accounts up to $50 \%$ of all abdominal TB 
cases [5]. However, there is no available epidemiological data of frequency of peritoneal tuberculosis among children due to its rarity. It is an infrequent manifestation of TB, especially in children without comorbidities [2,3]. Diagnosis of peritoneal tuberculosis remains a challenge to the physicians because of vague and non-specific clinical features or low yield of Mycobacterium culture or smear [3]. The peritoneal tuberculosis must be differentiated with other infectious, inflammatory bowel and malignant diseases because of its non-specific manifestation [3].

The aim of this study was to observe and summarize experience and struggles in diagnosing peritoneal TB in children in different paediatric centres. We think that this disease may be neglected and not thought about by paediatricians and paediatric surgeons, because of its rarity and uncommon presentation. Therefore, we reviewed our hospital's experience and performed a systematic literature's review of children's peritoneal TB cases in the last decade.

\section{Methods}

\section{Eligibility criteria for studies}

Only articles with final peritoneal TB diagnosis were included. If there was no possibility to exclude patients only with peritoneal TB, articles were considered as not eligible. Studies with data about patients' clinical presentation, test results, surgical appearance were considered eligible. Only cases with patients who were younger then 18 years of age at the time of diagnosis were included, if there was no possibility to exclude older patients, articles were not selected for further analysis. If the article's full-text was not available in English, it was excluded from the review.

\section{Systematic review: information sources, PubMed search analysis}

The separate cases reports and retrospective reviews of children with abdominal and peritoneal tuberculosis published from January 2008 to December 2019 were searched in the PubMed database. Two of the co-authors reviewed initial results independently. After excluding articles of adult patients, non-English language, non-full text articles and duplicates, 83 articles remained for further analysis (Fig. 1). Additionally, 40 articles from 83 were excluded because of other TB forms, 2 because of inability to exclude only peritoneal TB cases, 2 because TB was caused by Mycobacterium bovis, 22 articles because of lack of specific patient's data and in 6 articles patients older then 18 years old were included. The 11 case series (with 44 patients) were included for the final analysis (Fig. 1.).

In each study the data were extracted for the following outcomes: demographic features, symptoms, laboratory, radiological, microbiological and surgical findings. The time until hospitalisation was defined as the time from the presentation of the symptoms until attendance to the hospital. The time until diagnosis was set to be the time from the first day at the hospital until the final diagnosis. The histopathological test would be evaluated as positive if tuberculous granulomas were found. 


\section{Our experience}

In our centre, we had 4 cases of peritoneal TB during the period of 11 years. These patients' medical records were reviewed for the same data as for the systematic literature review. After that they were included in the statistical analysis.

Statistical analysis for systematic review was performed using SPSS version 23.0. Median with standard errors for age was calculated. White blood cells (WBC), erythrocytes sedimentation rate (ESR), $C$ reactive protein (CRP) levels, time until hospitalization and diagnosis were calculated as a mean value with standard deviations.

\section{Results}

\section{Our experience}

Clinical data of 4 children with peritoneal tuberculosis during the past 11 years in Lithuanian University of Health Sciences, Kaunas Clinics Pediatric Surgery department were collected and analyzed. Patients manifested unspecific symptoms such as abdominal pain, persistent fever, nausea and fatigue. After standard investigations such as blood test, CRP, urine analysis, abdominal ultrasound and X-ray the diagnosis remained unclear. After some additional examination, patients underwent diagnostic laparoscopy. Diagnosis of peritoneal tuberculosis was confirmed by peritoneum biopsy and histopathological examination. They recovered fully after anti-tuberculosis therapy. In the first 3 cases diagnosis was late, but in the 4th case it was made quicker because of the experience.

\section{The Demographic features and anamnesis}

Total number of 48 patients ( 44 from systematic review and 4 from our own experience) were included for the further analysis. $29(60.4 \%)$ patients were males with the median age of 11 (range 1.5-16) and 19 (39.6) patients were females with a median age 10 (range 2.5-17). The age of the patients was not adjusted for the gender in the two articles. The contact with the person of active pulmonary TB was reported in the 18 cases (37.5\%). Twenty-nine patients (60.4\%) were vaccinated with BCG vaccine $(p=$ 0.0487). Most of the patients $(72.9 \%)$ were followed-up for the final outcome. There were reported low complications rates after TB treatment ( 2 cases $(4.2 \%)$ ): one patient was reported to have pyrazinamideassociated thrombocytopenia and other patient presented with small bowel obstruction secondary to adhesions, requiring laparotomy 2 years after the initial diagnosis (table 1 ).

Table 1. Demographic features. 


\begin{tabular}{|c|c|c|c|c|c|c|}
\hline \multirow{5}{*}{$\begin{array}{l}\text { Demographic } \\
\text { features }\end{array}$} & \multicolumn{4}{|l|}{$P$ value } & \multirow{2}{*}{$\begin{array}{l}0.1492 \\
+18 \\
(37.5 \%)\end{array}$} & \multirow{2}{*}{$\begin{array}{l}0.0487 * \\
+29 \\
(60.4 \%)\end{array}$} \\
\hline & Summary & $\begin{array}{l}2008- \\
2018\end{array}$ & - & $\begin{array}{l}48(M=29 \\
60.4 \% ; F=19\end{array}$ & & \\
\hline & & & & & $\begin{array}{l}-27 \\
(56.3 \%)\end{array}$ & $-13(27.1 \%)$ \\
\hline & 12 & $\begin{array}{l}\text { Our } \\
\text { reports, } \\
2018\end{array}$ & $\begin{array}{l}\text { Lithuania } \\
\text { (4) }\end{array}$ & $4: 0$ & $3+$ & $4+$ \\
\hline & 11 & $\begin{array}{l}\text { Zaslavsky } \\
\text { J, } 2018\end{array}$ & $\begin{array}{l}\text { USA } \\
\text { (2) }\end{array}$ & $1: 1$ & $1+$ & \\
\hline
\end{tabular}

$\begin{array}{lll}\text { Ding DC, } & \text { Taiwan } & 0: 1 \\ 2010 & \end{array}$

(1)

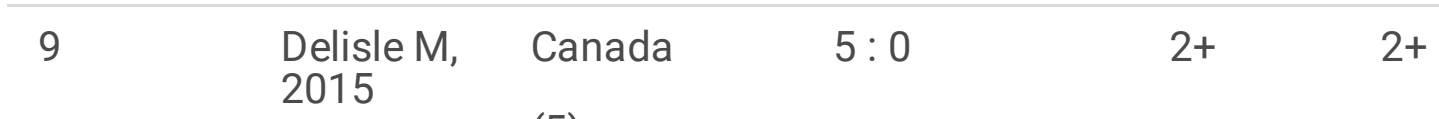

$\begin{array}{lllll}\text { Avcu G, } & \text { Turkey } & 0: 1 & 1- & 1+\end{array}$

(1)

\begin{tabular}{lllll}
\hline 7 & Patel RV, & UK & $1: 0$ & $1-$
\end{tabular}

(1)

\begin{tabular}{lllll}
\hline Menke J, & Germany & $0: 1$ & $1+$ \\
2013 & $(1)$ &
\end{tabular}

$5 \quad \begin{array}{lll}\text { Esposito } & \text { Italy } & 0: 1\end{array}$

(1)

\begin{tabular}{llllll}
\hline 4 & Pant N, & India & $9: 2$ & $4+$ & $7+$ \\
2011 & & & &
\end{tabular}

\begin{tabular}{|llllll|}
\hline 3 & $\begin{array}{l}\text { Al-Otaibi } \\
\text { A, 2011 }\end{array}$ & $\begin{array}{l}\text { Saudi Arabia } \\
(1)\end{array}$ & $0: 1$ & $1+$ & $1+$ \\
\hline 2 & $\begin{array}{l}\text { Chahed J, } \\
2010\end{array}$ & $\begin{array}{l}\text { Tunisia } \\
(11)\end{array}$ & $4: 7$ & $1+$ & $10+$ \\
\hline 1 & $\begin{array}{l}\text { Dinler G, } \\
2008\end{array}$ & $\begin{array}{l}\text { Turkey } \\
(9)\end{array}$ & $5: 4$ & $6+$ & $4+$ \\
\hline & $\begin{array}{l}\text { Author, } \\
\text { year }\end{array}$ & $\begin{array}{l}\text { Country } \\
\text { (number of } \\
\text { cases) }\end{array}$ & Patients M:F & $\begin{array}{l}\text { TB } \\
\text { contact }\end{array}$ & $\begin{array}{l}\text { BCG } \\
\text { vaccination }\end{array}$ \\
\hline
\end{tabular}




\section{'The clinical presentation and analysis' results}

The mean time from symptoms presentation until hospitalisation was $30.62 \pm 4.0$ days. Most of the patients presented with abdominal pain $(n=34,70.8 \%, p=0.0085)$, abdominal distention $(n=32,66.7 \%$, $p=0.0298)$ and fever $(n=26,54.2 \%)$. The ascites $(n=16,33.3 \%)$ and anorexia $(n=15,31.3 \%)$ manifested in one third of the cases. Other symptoms, such as nausea, vomiting, diarrhoea, fatigue, weight loss and night sweets were observed in less than $30 \%$ of all the cases (Table 2). The mean of WBC count was $8.8 \pm 1.2 * 10 / \mathrm{I}^{12}$, ESR $-56.3 \pm 10.2 \mathrm{~mm} / \mathrm{h}, \mathrm{CRP}-79.6 \pm 17.3 \mathrm{mg} / \mathrm{l}$.

Table 2

Clinical presentation of peritoneal tuberculosis

\begin{tabular}{|llll|}
\hline & Events/ total & Event rate, \% & P value \\
\hline Fever & $26 / 48$ & 54.2 & 0.0692 \\
\hline Abdominal pain & $34 / 48$ & 70.8 & $\mathbf{0 . 0 0 8 5 ^ { * }}$ \\
\hline Abdominal distention & $32 / 48$ & 66.7 & $\mathbf{0 . 0 2 9 8 ^ { * }}$ \\
\hline Anorexia & $15 / 48$ & 31.3 & 0.3831 \\
\hline Nausea & $6 / 48$ & 12.5 & $\mathbf{0 . 0 0 8 1 ^ { * }}$ \\
\hline Vomiting & $5 / 48$ & 10.4 & $\mathbf{0 . 0 2 4 6 *}$ \\
\hline Diarrhoea & $7 / 48$ & 14.6 & $\mathbf{0 . 0 2 4 0 *}$ \\
\hline Ascites & $16 / 48$ & 33.3 & 0.5266 \\
\hline Fatigue & $5 / 48$ & 10.4 & $\mathbf{0 . 0 1 8 8 ^ { * }}$ \\
\hline Weight loss & $14 / 48$ & 29.1 & 0.2656 \\
\hline Night sweets & $9 / 48$ & 18.7 & $\mathbf{0 . 0 4 1 4 ^ { * }}$ \\
\hline Comorbidities & $5 / 48$ & 10.4 & $\mathbf{0 . 0 1 8 8 ^ { * }}$ \\
\hline *p<0.05 & & & \\
\hline
\end{tabular}

The Tuberculin skin (Mantoux) test was performed in the majority of cases ( 42 cases, 87.5\%)), however, only half of them were positive $(n=21,43.7 \%)$. The QuantiFERON (QFT) test was performed for only 2 patients and it was positive in both cases. The Culture samples for $\mathrm{M}$. tuberculosis from sputum were taken in 13 cases $(27.1 \%)$ and only in $1(2.1 \%)$ came back positive. The culture samples from gastric aspirate was taken in 14 cases $(29.2 \%)$, but it was positive in 2 cases (4.2\%) only. Even though, the Culture sample from ascites was frequently taken $(n=31,64.6 \%$, it was negative in most of the cases $(n$ $=22,70.9 \%$ ) (Table 3). 
Table 3

Laboratory, microbiological, radiological and histopathological tests results

\begin{tabular}{|c|c|c|c|c|}
\hline & & Events/total & $\begin{array}{l}\text { Event rate, } \\
\%\end{array}$ & $\begin{array}{l}P \\
\text { value }\end{array}$ \\
\hline Tuberculin skin test & & $21 / 48$ & 43.7 & 1.000 \\
\hline Chest Xray/CT & & $16 / 48$ & 33.3 & 0.2434 \\
\hline Fibroesophagogastroduodenoscopy & & $1 / 48$ & 2.1 & 0.8877 \\
\hline \multirow{4}{*}{$\begin{array}{l}\text { Positive culture samples of } M \text {. } \\
\text { tuberculosis in }\end{array}$} & Sputum & 2.1 & $1 / 48$ & 0.6167 \\
\hline & $\begin{array}{l}\text { Gastric } \\
\text { aspirate }\end{array}$ & 4.2 & $2 / 48$ & 0.5266 \\
\hline & Ascites & 18.7 & $9 / 48$ & 0.1650 \\
\hline & Pleural liquid & 0 & $0 / 48$ & \\
\hline Biopsy of peritoneum & & $38 / 48$ & 79.2 & \\
\hline Tuberculous granulomas & & $38 / 38$ & 100 & \\
\hline
\end{tabular}

Radiological findings

Chest X-Ray or computed tomography (CT) was done for $85.4 \%(n=41)$ patients, but the changes were observed in the third of the cases (16 cases (33.3\%)). The most common findings were free fluid in the pelvic cavity $(n=34,70.8 \%, p=0.0085)$ and the enlarged mesenteric lymph nodes $(n=25,52.1 \%)$. Additionally, the interloop fluid collections ( $n=11,22.9 \%)$, dilated bowel loops $(n=10,20.8 \%)$ and thickening of intestine wall $(n=9,18.7 \%)$, peritoneum $(n=7,14.6 \%)$ and omental caking $(n=6,12.5 \%)$ were less frequent. Other findings such as masses $(n=4)$, hepatomegaly $(n=2)$ or omental caking $(n=6)$ were found in less than $10 \%$ of all the cases (Table 4 ). 
Table 4

UG/CT findings

\begin{tabular}{|llll|}
\hline & Events/total & Event rate, \% & P value \\
\hline Free fluid in pelvic cavity & $34 / 48$ & 70.8 & $\mathbf{0 . 0 0 8 5 *}$ \\
\hline Thickened peritoneum & $7 / 48$ & 14.6 & $\mathbf{0 . 0 0 0 1 *}$ \\
\hline Mass & $4 / 48$ & 8.2 & $<\mathbf{0 . 0 0 0 1 *}$ \\
\hline Enlarged mesenteric lymph nodes & $25 / 48$ & 52.1 & 0.7736 \\
\hline Thickening of small intestine wall & $9 / 48$ & 18.7 & $\mathbf{0 . 0 0 0 3 *}$ \\
\hline Dilated bowel loops & $10 / 48$ & 20.8 & $\mathbf{0 . 0 0 2 8}$ \\
\hline Interloop fluid collection & $11 / 48$ & 22.9 & $\mathbf{0 . 0 0 1 1 ^ { * }}$ \\
\hline Hepatomegaly & $2 / 48$ & 4.2 & $<\mathbf{0 . 0 0 0 1 *}$ \\
\hline Omental caking & $6 / 48$ & 12.5 & $<\mathbf{0 . 0 0 0 1 *}$ \\
\hline * $p<0.05$ & & & \\
\hline
\end{tabular}

\section{Surgical appearance}

A surgical intervention was performed in 36 (75\%) cases: 28 (77.7\%) laparoscopy, 4 of them with a conversion to laparotomy and $5(13.8 \%)$ laparotomy. The type of 3 surgical interventions were not further specified. Tubercles $(n=23,63.8 \%)$, peritonitis $(n=15,41.6 \%)$ and ascites $(n=13,36.1 \%)$ were the most common findings. During the operation other findings like adhesions $(n=10)$, thickened peritoneum $(n=$ 8), omental changes $(n=4)$, intestinal investment $(n=3)$ or enlarged lymph nodes involvement $(n=3)$ were revealed in less than $30 \%$ of all cases (Table 5 ). The histopathological biopsy of peritoneum was performed in the majority of patients $(n=38,79.2 \%)$ and tuberculous granulomas were notified in all of them $(100 \%)$. The mean time of from patients arrival to the hospital until diagnosis. The mean time until hospitalisation was $30.62 \pm 4.0$ days and the mean time until diagnosis was $17.4 \pm 3.7$ days. 
Table 5

Surgical appearance

\begin{tabular}{|llll|}
\hline & Events/total & Event rate, \% & P value \\
\hline Surgical intervention & $36 / 48$ & 75 & \\
\hline Ascites & $13 / 36$ & 36.1 & 0.2152 \\
\hline Tubercles & $23 / 36$ & 63.8 & 0.0738 \\
\hline Peritonitis & $15 / 36$ & 41.6 & 0.5262 \\
\hline Adhesions & $10 / 36$ & 27.7 & $\mathbf{0 . 0 4 0 6 *}$ \\
\hline Thickened peritoneum & $8 / 36$ & 22.2 & $\mathbf{0 . 0 1 2 7 *}$ \\
\hline Omental changes & $4 / 36$ & 11.1 & $\mathbf{0 . 0 0 2 1 *}$ \\
\hline Intestinal investment & $3 / 36$ & 8.3 & $\mathbf{0 . 0 0 1 7 *}$ \\
\hline Lymph nodes involvement & $3 / 36$ & 8.3 & $\mathbf{0 . 0 0 1 7 *}$ \\
\hline * $\mathrm{p}<0.05$ & & & \\
\hline
\end{tabular}

\section{Discussion}

Tuberculosis currently remains to be a major problem in undeveloped, as well as in developing countries with a significant cause of mortality. It is one of the top 10 causes of death worldwide and a leading cause from a single infection. Growing migration rates increase the risk of spreading tuberculosis from endemic countries. Furthermore, diagnosis of peritoneal TB is still challenging because of its unspecific manifestation. One of the WHO aims is strengthening data collection for children and adolescents with TB [6]. After our own complicated experience with 4 cases of peritoneal TB we searched for evidence based diagnostic guidelines, but we were not able to find any. As we know, there is no systematic literature review of children's peritoneal TB, possibly because of its rare incidence and the lack of cumulative data. There are only single cases reports with children's peritoneal TB reported from separated centres. These were the reasons why we conducted this systematic review and analysis. As our study shows, individual cases of peritoneal TB in children are observed in regions with a different morbidity of TB, social and economic status including experience of four different continents - most of cases presented from India (11), Tunisia (11) and Turkey (10) and others from Saudi Arabia, Italy, Germany, United Kingdom, Canada, Taiwan and United States of America [3, 4, 7-15].

Despite the fact that extrapulmonary TB is more common for patients with comorbidities, our review showed that only $10.4 \%$ of children had additional systemic diseases (AIDS, asthma, recurrent urinary tract infections, epilepsy, cerebral palsy). Other children were reported to be healthy and previously vaccinated with BCG vaccine (60.4\%). Although, detailed epidemiological anamnesis of possible contact with TB patient is extremely important, positive TB anamnesis was reported in only third of all cases. 
Therefore, if the patient can not disclose a previous contact with TB, it should not reduce the suspicion of extrapulmonary TB form for a child from a normal socioeconomic environment.

As previously reported, the clinical presentation of peritoneal TB in children is mostly subacute and various between weeks to months [7]. This review also confirms that the average time from the presentation of symptoms until the hospitalization was about one month, what regrettably leads to the delayed treatment of TB. Even though, the time from the patient's hospitalization until correct diagnosis of peritoneal TB diagnosis was not mentioned in most of the articles, we know that due to its unspecific manifestation it can be delayed as well. For instance, it took about 2 weeks to set the peritoneal TB diagnosis in our patients. Additionally, the peritoneal TB diagnosis for children is rarely suspected only upon clinical presentation [12]. As this study shows, the most common observed symptoms were unspecific and very common for every sick child: abdominal pain with distention and fever. Up to $10 \%$ of all children presented to the Emergency department complains of abdominal pain [16]. Moreover, fever is the most common complain of pediatric patients in emergency department [17]. Additionally, such symptoms as anorexia and ascites could be observed as well. Although more common diseases like appendicitis, infectious diseases or malignancies are thought about and diagnosed in most of the cases $[10,14]$. That is why children with such symptoms should be always suspected for possible TB, even though the child had no previous contacts with TB.

According to the literature, pleural or pulmonary involvement is reported in $15-75 \%$ of patients with extrapulmonary TB [19-21]. However, this systematic review revealed that most of the children with peritoneal TB presented with normal chest X-Rays or CT scans. Nevertheless, abdominal UG or CT were abnormal in the better part of the cases. The most common observed findings were free fluid in pelvic cavity and enlarged mesenteric lymph nodes that together with clinical presentation could alert for possible peritoneal TB diagnosis in children (Fig. 2). Although clinical practice demonstrates, these symptoms are quite common in cases of mesadenitis. Despite that peritoneal tuberculosis should not be forgotten.

Mantoux (tuberculin skin) test remains one of the best known first investigation tool that increases suspicion about TB [16]. Unfortunately, for half of the patients included in this review it was negative, that shows it is not enough specific test for peritoneal TB. Although, Quantiferon test, also known as interferon-y release assay, is reported to be more specific then tuberculin skin test [17], with the sensitivity of $93.1 \%$ and specificity $-76.7 \%$ [18], it was performed for only 2 patients.

In case of peritoneal TB, most of usually performed laboratory tests are non-specific [3]. Our systematic literature's review confirmed this argument, showing that the mean WBC and ESR does not exceed normal limits or were slightly elevated, however moderately elevated CRP was observed in most of the cases. The culture samples were not specific diagnostic tool as well. The culture samples for Mycobacterium tuberculosis are not so often accomplished in most of the countries. One of the reasons is complicated performance for children and often delayed results [19]. It is obvious from the review that when peritoneal TB is suspected, most culture samples taken from ascites, sputum, gastric aspirate and pleural liquid were negative. 
As literature shows, nowadays, the most specific laboratory test known for peritoneal TB is ascitic fluid analysis that includes serum/ascites albumin gradient (SAAG) test and adenosine deaminase activity (ADA) test with sensitivity of $100 \%$ and specificity of $97 \%$. It might help easily in differential diagnosis of ascites origin $[2,19]$. Unfortunately, it was mentioned only in a few cases included in this review, because not many centres have knowledge or possibility to do this test. There is a notion, that the gold standard for diagnosing peritoneal TB is laparoscopy with peritoneum biopsy. This systematic literature reveals that surgical intervention was performed in three quarters of all the cases. Tubercles, peritonitis and ascites were the most common surgical findings that lead to the suspicion of peritoneal TB diagnosis. Histopathological results of tuberculous granulomas from peritoneum biopsy was extremely specific and accurate ( $100 \%$ positive) for peritoneal tuberculosis as were the case in our 4 cases as well when the diagnosis of peritoneal TB was conducted only after the biopsy.

To our knowledge now, we speculate that surgery leads to unnecessary surgical risk, prolonged time until diagnosis, as well as prolonged hospitalization. Therefore, the ascitic fluid analysis, that includes serum/ascites albumin gradient (SAAG) test and adenosine deaminase activity (ADA) test in children would be way moreless invasive method to diagnose peritoneal TB then laparoscopy or laparotomy. Moreover, it can be mostly done under ultrasound (US) guidance and in older children it is even available under local anaesthesia.

Based on our own experience and systematic literature review of peritoneal TB, we suggest an algorithm for diagnosing suspected peritoneal TB that we included in clinical practise in our Hospital. It suggests that if patient is presented with abdominal pain, distension and fever, elevated CRP and ESR, with no or mild leucocytosis, US finds free fluid in pelvic cavity, enlarged mesenteric lymph nodes you should suspect peritoneal TB. This idea should be followed by Quantiferon test and then ascitic fluid analysis. Only if diagnosis is still unclear after these tests, laparoscopy with peritoneum biopsy should be performed (Fig. 2). Our center experience, as well as this systematic literature review shows culture samples has low specificity, prolongs the time until the diagnosis. Moreover, taking these samples are invasive procedures (bronchoscopy, endoscopy), in most of the cases requires anesthesia. This follows conclusion, there are more specific, quicker tests for peritoneal TB diagnosis.

\section{Limitations Of The Study}

First of all, the scientific quality of the data was generally poor because only 11 full text articles met the criteria for the final analysis. Secondly, only 1 database was searched due to our institution limited data sources. Additionally, in our study only cases of peritoneal tuberculosis were included in the study due to our centre experience. However, for the future analyses, not only peritoneal, but also all abdominal tuberculosis cases could be analysed .

\section{Conclusions}


For our knowledge, it is the first systematic literature review analysing children with peritoneal tuberculosis worldwide. As systematic review showed, clinical presentation, laboratory test results, culture samples, X-Ray, US and CT findings are unspecific for peritoneal TB diagnosis in children. Laparoscopy or laparotomy with peritoneum biopsy was performed in the majority of analysed cases $[9,11]$. Although, we suggest that before diagnostic surgery, Quantiferon test and ascitic fluid analysis with SAAG and ADA tests should be performed. In this way diagnosis might be confirmed without unnecessary surgery.

\section{Abbreviations}

ADA - adenosine deaminase activity test

BCG - bacilli Calmette-Guérin

CRP - $\mathrm{C}$ reactive protein

CT - computed tomography

ESR - erythrocytes sedimentation rate

QFT - QuantiFERON test

SAAG - serum/ascites albumin gradient test

US - ultrasound

TB - tuberculosis

WHO - World Health Organization

WBC - white blood cells

\section{Declarations}

Ethical approval was issued by the Ethics Committee of the Lithuanian University of Health Sciences (protocol No. BEC-MF-149). Written consent was obtained from all the patients or their representatives.

The datasets analyzed during the current study available from the corresponding author on reasonable request.

There was no conflict of interests.

There was no funding for this systematic literature review.

Acknowledgements - not applicable. 


\section{References}

1. Segar M, Reuters. WHO Global Tuberculosis Report Executive Summary 2018. 2018; Available from: http://www.who.int/tb/publications/global_report/tb18_ExecSum_web_40ct18.pdf.

2. World Health Organization. Global Tuberculosis. Vol. 312, Jama. 2017. 28-58 p.

3. Global tuberculosis report 2020. Geneva:World Health Organisation; 2020. Licence: CC BY-NC-SA 3.0 IGO.

4. Dinler G, Şensoy G, Helek D, Kalaycı AG. Tuberculous peritonitis in children: Report of nine patients and review of the literature. 2008;14(47):7235-9.

5. Wu DC, Averbukh LD, Wu GY. Review Article Diagnostic and Therapeutic Strategies for Peritoneal Tuberculosis. A Review. 2019;7:140-8.

6. Avcu G, Sensoy G, Karli A, Caltepe G, Sullu Y, Belet N, et al. SHORT REPORT A case of tuberculous peritonitis in childhood. J Infect Public Health. 2015;8(4):369-72. Available from: http://dx.doi.org/10.1016/j.jiph.2015.03.004.

7. Liberati A, Altman DG, Tetzlaff J, Mulrow C, Gøtzsche PC, loannidis JPA, et al. The PRISMA Statement for Reporting Systematic Reviews and Meta-Analyses of Studies That Evaluate Health Care Interventions: Explanation and Elaboration. 2009;6(7).

8. Information G. Modified Ashworth Scale Instructions Patient Instructions: 1987;1-3.

9. Fehér ÁM, Bajory Z. A review of main controversial aspects of acute testicular torsion. J Acute Dis. 2016;5(1):1-8. Available from:

http://www.sciencedirect.com/science/article/pii/S2221618915000785.

10. Chahed J, Mekki M, Mansour A, Brahim M, Ben, Maazoun K, Hidouri S, et al. Contribution of laparoscopy in the abdominal tuberculosis diagnosis: Retrospective study of about 11 cases. Pediatr Surg Int. 2010;26(4):413-8.

11. Pant N, Choudhury SR, Gupta A, Yadav PS, Grover JK, Chadha R. Umbilical signs of peritoneal tuberculosis in children. Indian J Pediatr. 2012;79(9):1192-6.

12. Patel RV, Jackson P, Zani A, De Coppi P. Laparoscopic management of midgut malrotation and tuberculous peritonitis in an adolescent boy. BMJ Case Rep. 2014;2013-5.

13. Ding DC, Chu TY. Laparoscopic diagnosis of tuberculous peritonitis mimicking ovarian malignancy. Taiwan J Obstet Gynecol. 2011;50(4):540-2. Available from:

http://dx.doi.org/10.1016/j.tjog.2011.10.028.

14. Zaslavsky J, Mulugeta-Gordon L, Vasko I, Presenza T, Scattergood E, Meislich D, et al. Tuberculous peritonitis in children: Two case reports highlighting the important role of imaging. Radiol Case Reports. 2018;13(4):862-6. Available from: https://doi.org/10.1016/j.radcr.2018.05.010.

15. Delisle M, Seguin J, Zeilinski D, Moore DL. Paediatric abdominal tuberculosis in developed countries: Case series and literature review. Arch Dis Child. 2016;101(3):253-8.

16. Esposito S, Bosis S, Canazza L, Tenconi R, Torricelli M, Principi N. Peritoneal tuberculosis due to multidrug-resistant Mycobacterium tuberculosis. Pediatr Int. 2013;55(2):2012-4. 
17. Al-Otaibi A, Almuneef M, Hameed T. An unusual combination of extrapulmonary manifestations of tuberculosis in a child. J Infect Public Health. 2012;5(2):203-6. Available from: http://dx.doi.org/10.1016/j.jiph.2011.11.005.

18. Harris BR, Chinta SS, Colvin R, Schnadower D, Tarr PI, Sayuk GS. Undifferentiated Abdominal Pain in Children Presenting to the Pediatric Emergency Department. 2019.

19. Wing R. Fever in the Pediatric Patient. Emerg Med Clin NA. 2020;31(4):1073-96. Available from: http://dx.doi.org/10.1016/j.emc.2013.07.006.

20. Cruz AT, Starke JR, Sendi P, Battegay M, Padley SPG, Ramanathan VD. Clinical manifestations of tuberculosis in children. Paediatr Respir Rev. 2007;8(2):107-17. Available from: http://www.ncbi.nlm.nih.gov/pubmed/17574154.

21. Manohar A, Simjee AE, Haffejee AA, Pettengell KE. Symptoms and investigative findings in 145 patients with tuberculous peritonitis diagnosed by peritoneoscopy and biopsy over a five year period. Gut. 1990;31(10):1130-2.

22. Rasheed S, Zinicola R, Watson D, Bajwa A, Mcdonald PJ. Intra-abdominal and gastrointestinal tuberculosis. Color Dis. 2007;9(9):773-83.

23. Drobac PC, Shin SS, Huamani P, Atwood S, Furin J, Franke MF, et al. Risk Factors for In-Hospital Mortality Among Children With Tuberculosis: The 25-Year Experience in Peru. Pediatrics. 2012;130(2):e373-9. Available from: http://pediatrics.aappublications.org/cgi/doi/10.1542/peds.2011-3048.

24. Readmissions H, Program R. HHS Public Access. 2016;131(20):1796-803.

25. Ge L, Ma JC, Han M, Li JL, Tian JH. Interferon-y release assay for the diagnosis of latent Mycobacterium tuberculosis infection in children younger than 5 years: A meta-analysis. Clin Pediatr (Phila). 2014;53(13):1255-63.

\section{Figures}




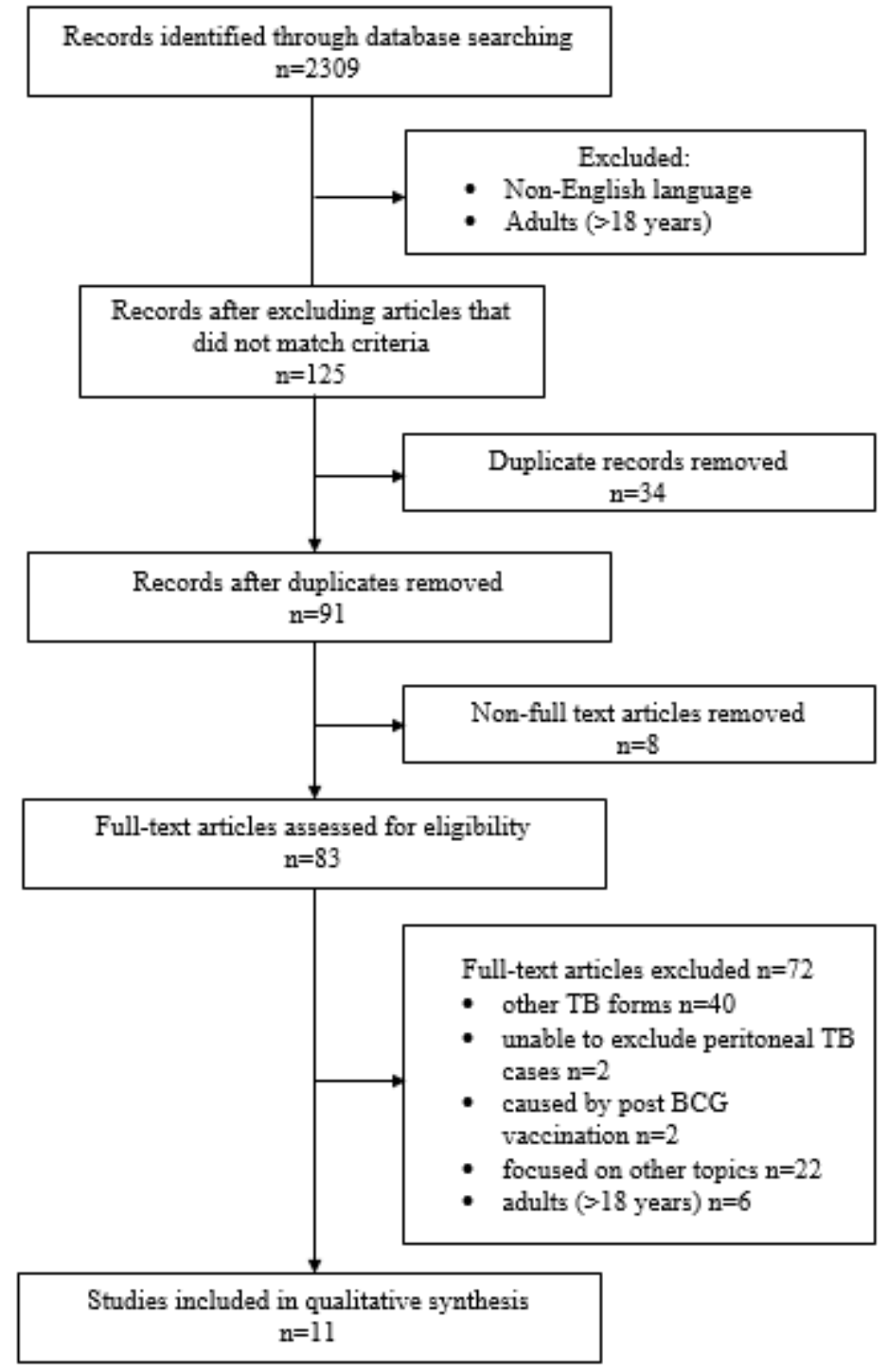

Figure 1

Additionally, 40 articles from 83 were excluded because of other TB forms, 2 because of inability to exclude only peritoneal TB cases, 2 because TB was caused by Mycobacterium bovis, 22 articles because of lack of specific patient's data and in 6 articles patients older then 18 years old were included. The 11 case series (with 44 patients) were included for the final analysis 


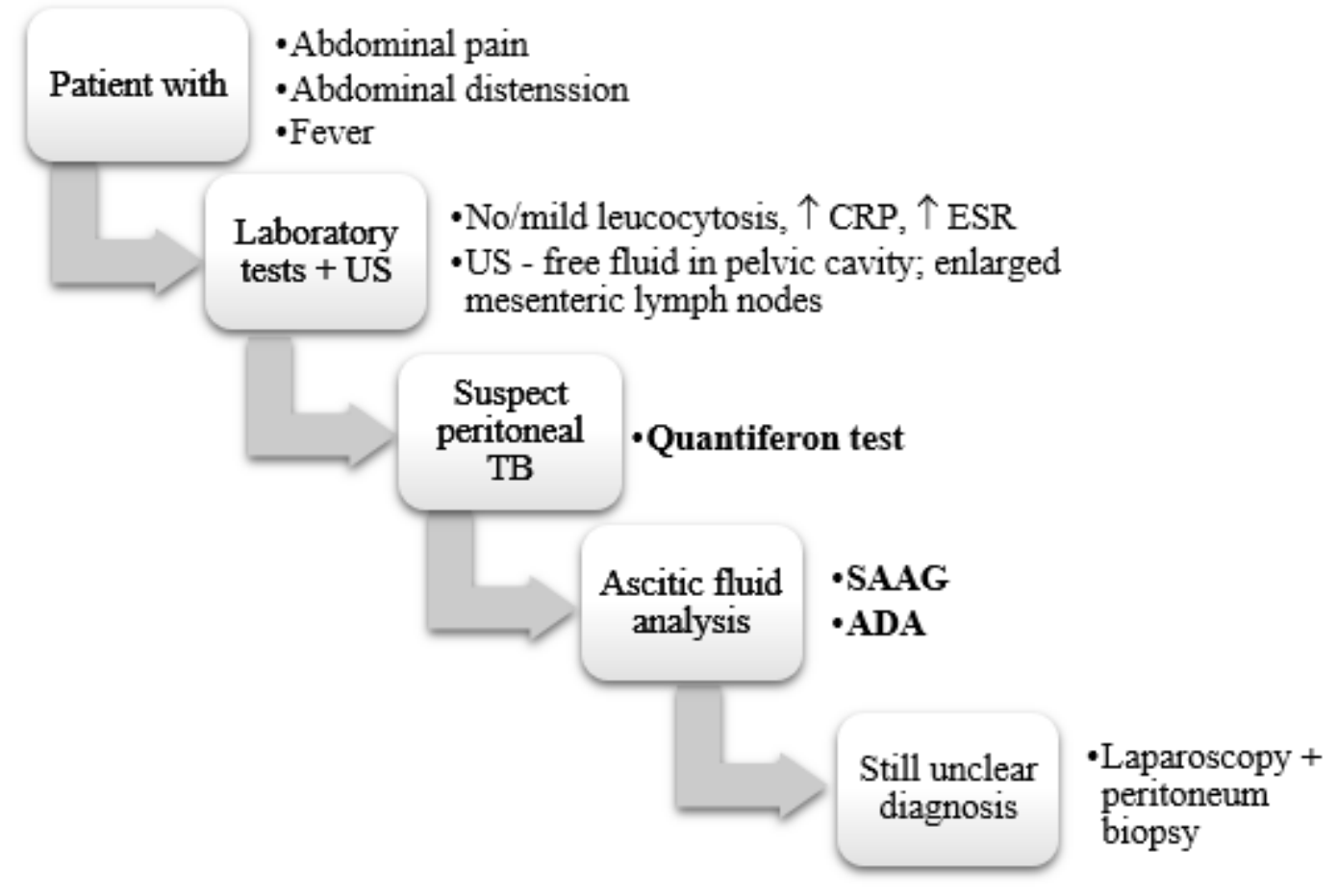

Figure 2

Our center experience, as well as this systematic literature review shows culture samples has low specificity, prolongs the time until the diagnosis. Moreover, taking these samples are invasive procedures (bronchoscopy, endoscopy), in most of the cases requires anesthesia. This follows conclusion, there are more specific, quicker tests for peritoneal TB diagnosis. 